\title{
Pathological Role of the "Invisible" Anastomoses
}

\section{Vladimir Ermoshkin*}

Russian New University (RosNOU), Moscow, Russia

*Corresponding author: Vladimir Ermoshkin, Russian New University (RosNOU), Moscow, Russia, Tel: 7-9645069550; E-mail : evlad48@list.ru

Received date: November 07, 2016; Accepted date: November 10, 2016; Published date: November 20, 2016

Copyright: (c) 2016 Ermoshkin V. This is an open-access article distributed under the terms of the Creative Commons Attribution License, which permits unrestricted use, distribution, and reproduction in any medium, provided the original author and source are credited.

\begin{abstract}
Thesis: An attempt to analyse the mechanism of cardiovascular disease (CVD). It is now believed that most heart disease of unknown etiologic and mechanism, with the CVD mortality is very high. Why theoretical medicine for over 100 years has such limited success? What is the true reason, for example, venous plethora?
\end{abstract}

Methods: Finding information in the literature, participation in medical conferences in Russia (2012-2016), as well as in Australia, Brisbane "Arrhythmia-2016", correspondence with scientists, discussions with leading Russian cardiologists, publication of my original articles.

Results: I was able to show that in modern theoretical cardiology there are significant errors. Our group with an instrument "Cardiocode" were able to confirm the presence of large arteriovenous anastomoses (AVA) in otherwise healthy people. The sudden opening of the lumen AVA causes a sharp drop in blood pressure.

When closing AVA, usually a few seconds, the blood pressure increases again. Such manipulations AVA, apparently provided by nature to protect the blood vessels from the peak pressure values. It turns out that the longterm "wrong" operation AVA, especially when an unhealthy lifestyle usually leads to two major problems.

First problem: The periodic disturbances of heart rhythm, sometimes with fatal outcome, due to excitation of the heart by means of a pulse of mechanical waves running through the contour of the vessels with the return of their heart. The second problem is: violation of "metabolism" of the prolonged increase in venous pressure as a systemic and local. This paper attempts to study the causes of venous plethora and diseases of the pelvic organs in humans.

Conclusions: I believe that, taking into account the vast experience of the modern official medicine, taking into account the large number of clinical observations, and using in the near future, the theory proposed by me might be able to make a breakthrough in the treatment of cardiovascular disease, including in the treatment of venous plethora.

Keywords: Arteriovenous anastomosis; Pelvic plethora syndrome; Pelvic pain syndrome; Arrhythmia

\section{Introduction}

Currently, the problem of prevention and treatment of cardiovascular disease is very serious. Why the cause of heart disease and sudden cardiac death (SCD) was now so relevant? Why cardiologist's theorists have no Nobel Prize for 116 years? Prize of Cardiology have only 2 people. The first prize winner in 1924 has become a specialist in the field of chemistry and pharmacy Einthoven, who in 1924 received an award for its old, made back in 1887 for the invention of the ECG. The second prize winner was the expert of the company "Herbalife" Luis Navarro, who in 1998 described the role of nitric oxide as a signalling molecule in the regulation of the cardiovascular system. In this way, advances in theoretical cardiology for such a long period of low. For example, in modern medicine, it was thought that the problem of cardiac arrhythmia is hidden at the micro level: in the micro and macro re-entry, in the ectopic foci. But according to my new theory of the nature of rhythm irregularities - is open anastomoses AVA overflow vena cava and the concentration of the pulse of mechanical waves at the myocardium. In the course of further research it appears that the increased venous pressure quickly leads to much cardiovascular disease.

This article focuses primarily on the problem of venous plethora as one of the most important at the present stage.

\section{Results}

Some of cardiology doubt that the work of the small "invisible" arteriovenous anastomoses (AVA) can lead to many diseases. But I always have the following question to those opponents: "What is the mechanism, net of the effect of hydrostatic pressure can increase the venous pressure in the portal vein to the critical level of $200-400 \mathrm{~mm}$ $\mathrm{H}_{2} \mathrm{O}$ and above?"After all, the human body has no other more powerful mechanisms to increase the pressure but the pressure generated by the aorta. In this normal pressure in venules in a healthy person should only be $70-130 \mathrm{~mm} \mathrm{H}_{2} \mathrm{O}$ ( $5.10 \mathrm{~mm}$ or $\mathrm{Hg}$ ).

AVA can be opened and closed, and these manipulations lead to pressure surges. As a rule, it reduces blood pressure and increases venous. These phenomena have been confirmed to device "CARDIOCODE" [1,2]. There is the observation that most working AVA appear in the vessels near the liver $[3,4]$. Yawning AVA even with 
the minimum aperture can lead to increased venous pressure in many organs, including pelvic organs.

Usually problems with AVA occur in people with a sedentary lifestyle, low physical activity. They also occur in people suffering from life is very big nervous or physical stress. When you open the AVA with the arterial blood pressure of about $30-60 \mathrm{~mm} \mathrm{Hg}$ It flows into veins, mixed with venous and moves generally upwardly in the direction of the right atrium by the inferior vena cava. The volume of the arterial blood decreases and increases venous. Mixed blood after passage AVA also moves toward the small veins, i.e., retrogradely in the direction where fluid pressure must be much smaller (about 5-10 $\mathrm{mmHg}$ ), i.e., in small veins of some organs. Of course, these two streams are not comparable in terms of volume of the pumped liquid. Flow towards venules and small veins (generally downward) are much smaller than in a continuous flow towards the heart (up the vena cava). Strictly speaking, the flow in the direction of the flow of small waves can be called only conditionally, in fact, created a blocking leakage of fluid pressure in the fabric of space crowded veins. These events lead to the effect of "hydraulic lock", primarily in the organs, located below the AVA in the pelvic organs and lower extremities, and the least in the lungs, as AVA light located above, at the same level with the heart. Note that the bodies blocked circulation temperature after a certain time is reduced. The blocked section of the wall of the small and medium-sized veins is stretched. These vessels even with venous valves are not capable of withstanding high pressure, so the liquid sooner or later runs into the extracellular space, and even occupies the free space between the bodies. There are cardiac arrhythmia due to run the pulse wave on the vena cava, and a mechanical pulse effects on the myocardium, except you experience swelling, stagnation, weight gain, "heart failure", varices, ascites [5-7]. On the other hand, due to the reduced at the open AVA gradient pressure between arterioles and venules occurs deceleration capillary circulation, cells do not receive power, stagnant blood becomes thicker lymph movement also slows critical drop strength immunity in stagnant fluid activated viruses and parasites. Note that the retrograde motion of mixed blood through the veins is precisely the laws of physics, and not according to the laws of human physiology. The forces of nature cannot cope with the growing problems of venous though night's sleep in a horizontal position weakens some swelling and stagnation. Nevertheless, within a few years of a person, if it is not treated and lead an unhealthy lifestyle can quite quickly be several cardiovascular and related diseases. There is so-called co-morbidity of diseases.

So, is now a topical issue in medicine venous plethora in the pelvic organs?

Yes it is. The official medicine there is pelvic congestion (hyperemia) syndrome, pelvic pain [8-10]. This problem is quite common, but specific numbers prevalence of pelvic congestion syndrome no. In view of the manifold manifestations of this syndrome in women is often masked by a variety of diagnoses: endometriosis, inflammatory diseases of appendages (adneksitomi), cystitis (bladder infection), intestinal diseases, etc. According to some estimates, up to $30-50 \%$ of women aged 20 to 50 years old have the syndrome, although not all of them have the characteristic symptoms.

It should be noted here that the skin, tendons, fascia and muscles in men are more durable, less stretch, the muscles of men carry a large amount of physical work, and so the problem of venous plethora in men is not as acute as in women. However, the value of pelvic congestion cannot be underestimated, for example in the development of diseases of the genito-urinary apparatus of men, prostatitis, and chronic hemorrhoids.

The problem is very serious, but the primary causes of pelvic congestion are not known. Official medicine believes that the development of venous plethora genetically predetermined. So, repeatability pelvic congestion from generation to generation is set to $50-70 \%$ of cases. Heritage in primarily two factors: the ability of the venous wall in significant tension and insufficient equipment venous valves, with their inherent anatomical deficiency. A role in the development of pelvic congestion has a hormonal influence, raising the level of estrogen, which in turn contribute to the expansion of the diameter of the pelvic veins.

Now, with the new knowledge about the causes of venous plethora of causes arrhythmia, a pathological role anastomoses AVA, apparently, in the near future we can expect a breakthrough in the understanding of the etiology of many cardiovascular diseases, among the many problems associated with the increased venous pressure [11-13].

But there are other problems such as the problem of finding a place AVA arrangement, the problem of monitoring the functioning incorrectly AVA, the problem of what to do and how to treat long gaping AVA. Recall that the open AVA helps reduce blood pressure and pathologically elevated venous pressure, which sooner or later leads to so many intractable diseases.

\section{Conclusion}

1. It appears that one of the major open secrets in medicine. It turns out much common cardiovascular disease it appears to have the same cause and the development of a similar mechanism.

2. The main cause of many cardiovascular diseases is open and for a long time not to close the arteriovenous anastomoses (AVA), which leads to blockage of blood flow in certain organs and stagnation of venous blood, especially in the legs and in the area of the pelvis. There is a syndrome of pelvic congestion (hyperemia), and pelvic pain syndrome.

3. In my opinion, the new theory of CVD found and continues to find a logical interpretation not only of individual clinical cases of cardiovascular disease, but also many related diseases that were previously considered separate nosological units.

4. The situation occurring in the cardiovascular system with open anastomosis (AVA) is similar to a ball pump with a small hole in it. In this case, the ball would never be quite inflated. The same is true with the heart when parted AVA. In such cases, the heart emits a sufficient amount of blood into the aorta, vena cava flow in often enough, too, the heart works too, but the irrational part of the blood (usually thicker) continues to be not involved in the systemic circulation and be out of the flow in the peripheral veins. It harms the body. The volume of arterial blood drops to a critical level, and the volume of venous excessive increases.

5. Apparently, the problem of finding and "treatment" open anastomoses AVA is very difficult. Here you need special study.

6. In my opinion, it is necessary to organize a series of "round tables", experiments to confirm and expand the new theory, begin to develop new treatment methods. The author of this report is ready to cooperate. Take care of yourself! 
Citation: Ermoshkin V (2016) Pathological Role of the "Invisible" Anastomoses. J Bioengineer \& Biomedical Sci 6: 209. doi:

Page 3 of 3

\section{References}

1. Lukyanchenko VA (2016) Cardiometric signs of performance of arteriovenous anastomosis in human cardiovascular system. Cardiometry. pp: 22-25.

2. Lukyanchenko VA (2016) Mechanisms of high heart rate variability: a fresh look. Cardiometry . pp: 26-30.

3. http://valsalva.ru/viewtopic.php? $\mathrm{t}=1101$ \&sid=137874936ec435e6be6626bf749f6a0f

4. Ermoshkin VI (2015) A new theory of arrhythmia, clarifies the causes of arrhythmias and some "metabolic disorders. Journal of Health and Education in the 21st Century.

5. Ermoshkin VI (2016) New theory of arrhythmia. Conceptual substantiation of arrhythmia mechanisms. Cardiometry. pp: 6-17.

6. Ermoshkin VI (2016) Heart transplantation mysteriously eliminates arrhythmia. Cardiometry. pp: 18-21.
7. Kamkin AG, Kiseleva IS, Yarygin VN (2002) Fibrillation, defibrillation. Priroda [Russian] 4: 1040.

8. Barkagan ZS (1980) Hemorrhagic diseases and syndromes M, Pelvic pain, causes of pain treatment in Kaluga, syndrome pelvic venous plethora. Pain Clinic.

9. Danilov AB (2016) Chronic pelvic pain - that it is important to know the neurologist.

10. Repin Danilov VV, Vorobyov Fateeva VV (2011) Chronic pelvic pain that it is important to know the neurologist. Breast cancer.

11. Ermoshkin VI (2016) New hypothesis of cardiac arrhythmias in humans. Cardiostim 40: 73 .

12. Ermoshkin VI (2016) Proceedings of the International Conference on arrhythmia [Abstract]. Australia, Brisbane: Arrhythmia.

13. Ermoshkin VI (2016) Arteriovenous anastomoses and cardiovascular diseases. 8th Cardiovascular Nursing and Nurse Practitioners Meeting. J Clin Exp Cardiology 7: 77. 